\title{
Reduction in ulnar pressure distribution when walking with forearm crutches with a novel cuff design: cross-sectional intervention study on the biomechanical efficacy of an ulnar recess
}

\author{
${ }^{1}$ Pascal Molteni, M.med.* \\ ${ }^{2}$ Thomas Hügle, M.D., Ph.D.* \\ ${ }^{3}$ Maria Hügle, B.Sc. \\ ${ }^{1,4}$ Corina Nüesch, Ph.D. \\ ${ }^{1,4}$ Annegret Mündermann, Ph.D.
}

${ }^{1}$ Clinic for Orthopaedics and Traumatology, University Hospital Basel, Spitalstrasse 21, 4031

Basel, Switzerland

${ }^{2}$ Department of Rheumatology, University Hospital Basel, Spitalstrasse 21, 4031 Basel, Switzerland

${ }^{3}$ Department of Computer Science, University of Freiburg, Georges-Köhler-Allee 51, 79110

Freiburg im Breisgau, Germany

${ }^{4}$ Department of Biomedical Engineering, University of Basel, Switzerland, Gewerbestr. 14, Allschwil, Switzerland

Molteni P., T. Hügle, M. Hügle, C. Nüesch, and A. Mündermann (2016) Reduction in ulnar pressure distribution when walking with forearm crutches with a novel cuff design: crosssectional intervention study on the biomechanical efficacy of an ulnar recess. Assistive Technology 2016 Oct 7. [Epub ahead of print]; doi 10.1080/10400435.2016.1236045.

Address for correspondence: PD Dr. Annegret Mündermann

Clinic for Orthopaedics and Traumatology

University Hospital Basel

Spitalstrasse 21

4031 Basel, Switzerland

Email annegret.muendermann@unibas.ch

Tel. +41613285445

Fax +41612657809

\section{Acknowledgements}

Rebotec for providing the Ulnar Pro cuffs. Rebotec did not have any input into the study design, analysis or interpretation.

Disclosures: Dr. Thomas Hügle designed the anatomically shaped cuff used in this study and receives royalties from the manufacturer. All other authors have no conflict of interest.

*shared first authors 


\begin{abstract}
Walking with crutches is an effective way of reducing the load on the lower extremity and is often indicated after injury or surgery. However, walking with forearm crutches with conventional cuffs can trigger symptoms including tenosynovitis in the biceps tendon, ulnar neuropraxia at the wrist, pain or skin hematoma. The purpose of this study was to test the hypothesis that a crutch cuff design with an ulnar recess reduces ulnar pressure during walking with forearm crutches. The pressure distribution between the forearm and crutch cuff was measured in 15 healthy participants for crutch walking with conventional and novel cuffs, respectively. Relative peak pressure in the proximal medial region compared to the overall peak pressure was reduced by $8.6 \%$ when walking with crutches with the novel cuff design compared to conventional cuffs $(\mathrm{P}<.001)$. Relative peak pressure in the distal intermediate and lateral regions were increased by $3.3 \%$ and $3.7 \%$ for the novel compared with conventional cuffs, respectively ( $\mathrm{P}<.001$ for both). Hence, the novel crutch cuffs shifted regions of high pressure away from the proximal ulnar region towards more distal regions that are covered by more soft tissue.
\end{abstract}

Key words: crutch walking, ulnar recess, pressure distribution, center of pressure 


\section{Background}

Walking with crutches is an effective way of reducing the load on the lower extremity and is often indicated after injury or surgery. However, increasing numbers of persons rely on crutches because age-related degenerative processes or diseases such as osteoarthritis, spinal stenosis or disability also increase (Vos et al., 2013). While in the United States, axillary crutches are most frequently used, forearm crutches are common in Europe, and hence our research focuses on forearm crutches.

While facilitating lower ambulatory load at the joints of the lower extremity, walking with crutches comes at the cost of greater loads at the joints of the upper extremity. For instance, the glenohumeral joint may be loaded by more than $100 \%$ body weight during crutch gait with on leg completely unloaded with forces (Westerhoff et al., 2012)—for forearm crutches being even higher than for axillary crutches-whereas this joint is not loaded in normal ambulation. In addition, walking with forearm crutches can trigger tenosynovitis in the biceps tendon (Kruger \& Bischof-Leger, 2008) and cause ulnar neuropraxia at the wrist (Ginanneschi, Filippou, Milani, Biasella, \& Rossi, 2009) and at the forearm (Malkan, 1992) because of the contact of the hand and forearm with the handle and cuff of forearm crutches, respectively. Other complications such as pain or skin hematomamostly along the ulnar bone - and even ulnar bone fracture during crutch gait have been reported (McGoldrick \& O'Brien, 1988; Venkatanarasimha, Kamath, Kambouroglou, \& Ostlere, 2009).

Clinical complaints experienced when walking with forearm crutches can be alleviated by the specific design of the crutch components. For instance, Sala et al. (Sala, Leva, Kummer, \& Grant, 1998) have shown that crutch handle design influences palmar 
pressure distribution. It reduces pressure loads in specific anatomic regions and increases the load-bearing area on the palmar surface of the hands during ambulation. A recent study (Fischer et al., 2014) has shown that the pressure load on the forearm during walking with forearm crutches is located predominantly over the ulna. Moreover, mean pressure loads were related to forearm girth. These findings and the clinical observations have led to the development of a novel crutch cuff design with a recess over the ulna with the goal of reducing the pressure load on the ulna and hence presumably reduce complications of forearm crutch use. The purpose of this study was to test the hypothesis that a crutch cuff design with an ulnar recess reduces ulnar pressure during walking with forearm crutches.

\section{Methods}

Fifteen healthy volunteers ( 8 male; age, $46.4 \pm 14.0$ years; height, $1.76 \pm 0.11 \mathrm{~m}$; body mass, $72.6 \pm 10.0 \mathrm{~kg}$; body mass index, $23.6 \pm 2.1 \mathrm{~kg} / \mathrm{m}^{2}$ ) participated in this study after providing informed consent. None of the participants had any orthopedic injuries within the preceding twelve months. Six participants had previously used crutches because of an injury. The study was conducted in accordance with the declaration of Helsinki.

Each participant performed walking trials with a set of standard forearm crutches (Comfort-Soft, Rebotec, Quakenbrück, Germany). The crutches were equipped with anatomically shaped grips and with either standard forearm cuffs (Comfort-Soft anatomic cuffs, Rebotec, Quakenbrück, Germany) or forearm cuffs with an ulnar recess (Ulnar Pro anatomic cuffs, Quakenbrück, Germany; Figure 1). Both cuff types were length adjustable. The length from the olecranon to the styloid process of the ulna and the circumference of the forearm at the level of the proximal end of the cuff were measured using a tape measure. An experienced physiotherapist ensured the correct crutch configuration and position. 
Pressure distributions were measured using a pressure sensor array (Sensor Model 5101; Tekscan Inc., South Boston, USA; $11.8 \times 11.8 \mathrm{~cm} ; 15.5$ sensel per $\mathrm{cm}^{2} ; 120$ frames per second). Prior to each data collection session, the sensor arrays were calibrated using a 2point calibration procedure and by applying a known static weight according to the manufacturer guidelines. Sensors were aligned to a line $1 \mathrm{~cm}$ distal of the proximal rim of the cuff and in mediolateral direction centered to the cuff, and attached to the inside of the each right cuff using double sided tape. The sensor array was attached to the scanning electronics box that was connected to a computer via a $4.5 \mathrm{~m}$ USB cable. The box was attached to the upper arm using self-adhesive stretchable wrap bandage (GazoFix ${ }^{\circledR}$, BSN medical GmbH, Hamburg, Germany). This set up had good to moderate reliability for measuring pressure distribution between the forearm and crutch cuff for crutch walking (Fischer et al., 2014).

Participants were given sufficient time to familiarize with each of the two crutch cuff designs. Subsequently, participants performed three walking trials with at least three right forearm loadings with each cuff design. Participants covered the same distance during the recording time of all walking trials for both conditions. The order of conditions was randomized, and subjects were blinded to the condition. We chose 3-point crutch walking while completely unloading the right leg defined as starting in unilateral left leg stance, advancing both crutches, then advancing the left lower extremity while bearing the body weight on the crutches. Data for five crutch gait trials with at least three right forearm loadings each were recorded. 


\section{Data processing}

The pressure sensor array was divided into eight octants (proximal and distal into medial, intermediate, ulnar and lateral, respectively; Figure 2). The proximal ulnar octant corresponded to the main contact area of the ulna (Fischer et al., 2014). Each region measured $5.6 \mathrm{~cm} \times 2.8 \mathrm{~cm}$ (Figure 2). For each experiment and participant, the recorded forearm pressure values were used to calculate the average and peak pressures for each octant and for the entire sensor for each step. Because of the limitations of the sensors in measuring absolute pressures and because the outcome parameters was the pressure distribution, relative peak pressure for each octant was calculated as the percentage of the peak pressure of the entire sensor. The center of pressure was calculated for each frame, and the path of the center of pressure was obtained for each step. In addition, the pressure data within each octant was summed for visualizing total pressure load. Ellipses were fitted to the center of pressure points for each walking trial using a least square fitting routine written in MATLAB (The MathWorks Inc., Natick, MA, USA).

\section{Statistical analysis}

All statistical tests were carried out in SPSS Version 21 (IBM Corporation, Amonk, NY, USA). Data are represented as means \pm one standard deviation. Significant differences between conditions were detected using linear mixed models with crutch design as withinsubject factor and forearm circumference as between-subject factor followed by Bonferroni's multiple comparison test. The significance level for all statistical tests was set a priori to .006 .

\section{Results}

Relative peak pressure in the proximal medial region was reduced by $8.6 \%$ when walking with crutches with the novel cuff design compared to conventional cuffs $(\mathrm{P}<.001$; 
Figure 3). Relative peak pressure in the distal intermediate and lateral regions were increased by $3.3 \%$ and $3.7 \%$ for the novel compared with conventional cuffs, respectively ( $\mathrm{P}<.001$ for both). Changes in relative peak pressure in the other regions were small $(<2.5 \%)$ and not statistically significant.

The center of pressure points were located more medial during walking with novel compared with conventional cuffs in all subjects. On average, the center of pressure points during walking with novel cuffs were located $7 \mathrm{~mm}$ more medial compared with conventional cuffs $(\mathrm{P}<.001$; Figure 4$)$. The largest medial shift observed with the novel cuff was $19 \mathrm{~mm}$. The mean proximal-distal position, principle radii and orientation of the ellipse least square fitted to the center of pressure points remained unchanged between conditions $(\mathrm{P}$ $>$.124). Similarly, the accumulated pressure for each sensor moved away from the proximal aspect of the ulna to areas overlying the extensor carpi ulnaris and digitorum muscles (Figure 5). Forearm circumference did not significantly influence the effects of crutch cuff design on pressure distribution.

\section{Discussion}

The purpose of this study was to test the hypothesis that a crutch cuff design with an ulnar recess reduces ulnar pressure during walking with forearm crutches. The results of this study showed that such a novel cuff changes the pressure distribution between the cuff and forearm compared with conventional cuffs. More specifically, regions of high pressure shifted away from the proximal ulnar region towards more distal regions. Hence, these results confirmed our hypothesis. 
Interestingly, only the location of the center of pressure points changed when walking with crutches with the novel design but not size of the area where these center of pressure points can be found. These results suggest that the size of the region containing the path of the center of pressure is primarily determined by the task at hand which was the walking pattern. Hence, because of the given task of 3-point crutch walking without loading the right leg, participants seemed to not have altered their general gait pattern although we did not measure kinematics in this study. These observations suggest that the ulnar recess indeed unloaded the region at the proximal ulna that is prone to overuse injuries for crutch walking without affecting the task of crutch walking. These results are further supported by the fact that participants required little time for adjusting their gait to the novel cuff design and provided qualitative feedback of lower pressure in the proximal ulna region.

The reduction in peak pressure in the ulna region was more than two-fold of the increase in peak pressure in other regions. Moreover, increased relative peak pressures were observed in the approximate regions of the extensor carpi ulnaris and digitorum muscles, and hence presumably would not cause problems because high pressures are better dissipated by soft tissue than by bone. Hence, the results of this study suggest that symptoms of pain or ulcers overlying the proximal ulna experienced during prolonged crutch walking may be alleviated by the use of a novel cuff design with an ulnar recess without causing problems in other regions of forearm-cuff contact.

In this study, we tested a small group of healthy participants during crutch walking. Although most of our participants had previous experience with crutches, we did not include long-term users of crutches. However, results of a previous clinical study using the same cuff design (Hügle, Arnieri, Bünter, Schären, \& Mündermann, 2015) showed large improvements 
in comfort and reductions in pain in long-term users of crutches where participants of that study chose to keep the crutches with the novel cuffs upon completion of the study. Other factors such as handle design have been shown to affect crutch gait (Sala et al., 1998). However, in our study we only changed the crutch cuffs, and hence this factor did not influence our results.

\section{Conclusions}

We showed that novel crutch cuffs shift regions of high pressure between the cuff and forearm during crutch walking away from the proximal ulnar region towards more distal regions. The fact that these regions are covered by more soft tissue explain results of a previous clinical study of greater comfort and lower pain when walking with crutches with a cuff with ulnar recess in long-term crutch users. This novel crutch design represents a simple and low-cost solution to symptoms experienced by many short- to long-term crutch users that can be combined with other crutch components such as anatomic handles or shock absorbing tips.

\section{References}

Fischer, J., Nuesch, C., Gopfert, B., Mundermann, A., Valderrabano, V., \& Hugle, T. (2014). Forearm pressure distribution during ambulation with elbow crutches: a crosssectional study. Journal of Neuroengineering and Rehabilitation, 11(1), 61.

Ginanneschi, F., Filippou, G., Milani, P., Biasella, A., \& Rossi, A. (2009). Ulnar nerve compression neuropathy at Guyon's canal caused by crutch walking: case report with ultrasonographic nerve imaging. Arch Phys Med Rehabil, 90(3), 522-524. 
Hügle, T., Arnieri, A., Bünter, M., Schären, S., \& Mündermann, A. (2015). Prospective clinical evaluation of a novel anatomic cuff for forearm crutches. Swiss Medical Weekly, 145(Suppl 210), P84.

Kruger, M., \& Bischof-Leger, E. (2008). [Frequency of biceps tendon tenosynovitis from crutches: a sonographical observation]. Z Rheumatol, 67(1), 62, 64-67.

Malkan, D. H. (1992). Bilateral ulnar neuropraxia: a complication of elbow crutches. Injury, 23(6), 426.

McGoldrick, F., \& O'Brien, T. M. (1988). Bilateral stress fractures of the ulna. Injury, 19(5), $360-361$.

Sala, D. A., Leva, L. M., Kummer, F. J., \& Grant, A. D. (1998). Crutch handle design: effect on palmar loads during ambulation. Archives of Physical Medicine and Rehabilitation, 79(11), 1473-1476.

Venkatanarasimha, N., Kamath, S., Kambouroglou, G., \& Ostlere, S. J. (2009). Proximal ulna stress fracture and stress reaction of the proximal radius associated with the use of crutches: a case report and literature review. Journal of Orthopaedics and Traumatology, 10(3), 155-157.

Vos, T., Flaxman, A. D., Naghavi, M., Lozano, R., Michaud, C., Ezzati, M., ..., Murray, C. J. (2013). Years lived with disability (YLDs) for 1160 sequelae of 289 diseases and injuries 1990-2010: a systematic analysis for the Global Burden of Disease Study 2010. Lancet, 380(9859), 2163-2196.

Westerhoff, P., Graichen, F., Bender, A., Halder, A., Beier, A., Rohlmann, A., \& Bergmann, G. (2012). In vivo measurement of shoulder joint loads during walking with crutches. Clinical Biomechanics, 27(7), 711-718. 


\section{Figure legends}

Figure 1. Photograph of the Ulnar Pro ${ }^{\circledR}$ crutch cuff (left) and the cuff attached to the crutch with anatomic handle (right).

Figure 2. Illustration of the inside of the crutch cuff, the location of the ulnar recess in the Ulnar Pro ${ }^{\circledR}$ cuff and the location of the pressure sensor matrix split into eight octants.

Figure 3. Mean (1 standard deviation) relative peak pressures for walking with crutches with a standard cuff (black) and with crutches with the Ulnar Pro ${ }^{\circledR}$ cuff (grey) for each of the eight octants. ${ }^{*}$ significant difference between cuff type $(\mathrm{P}<.05)$.

Figure 4. Center of pressure points on the crutch cuff during walking trials with forearm crutches with a conventional cuff (black) and a cuff with ulnar recess (grey; silhouette of the recess indicated by dashed lines).

Figure 5. Accumulated pressure for each sensor for crutch walking with a conventional cuff (left) and with the novel cuff (right) for the same subject as Figure 4. Please note that the largest pressures values moved away from the proximal aspect of the ulna to areas overlying the extensor carpi ulnaris and digitorum muscles. 


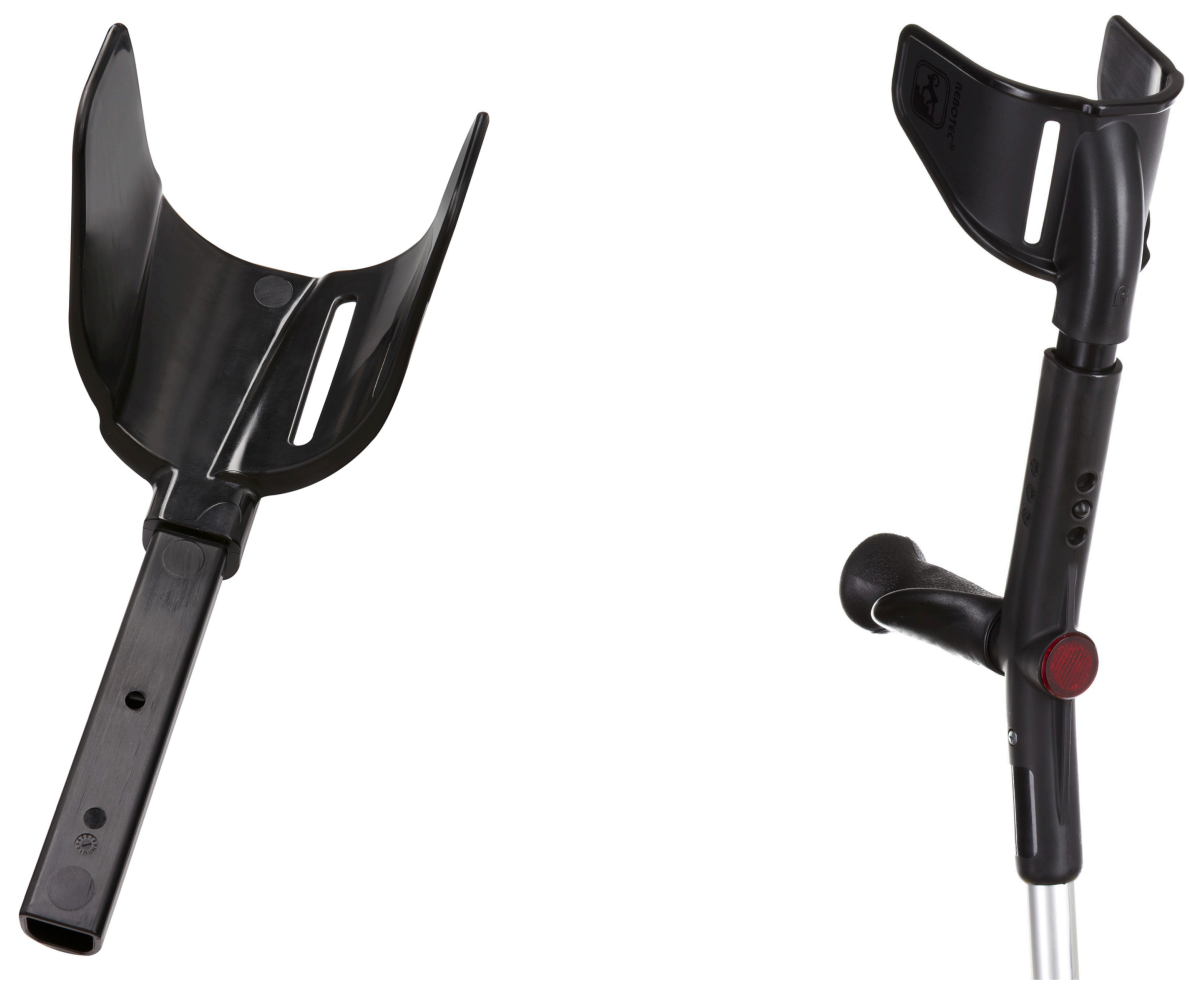

Figure 1

12 


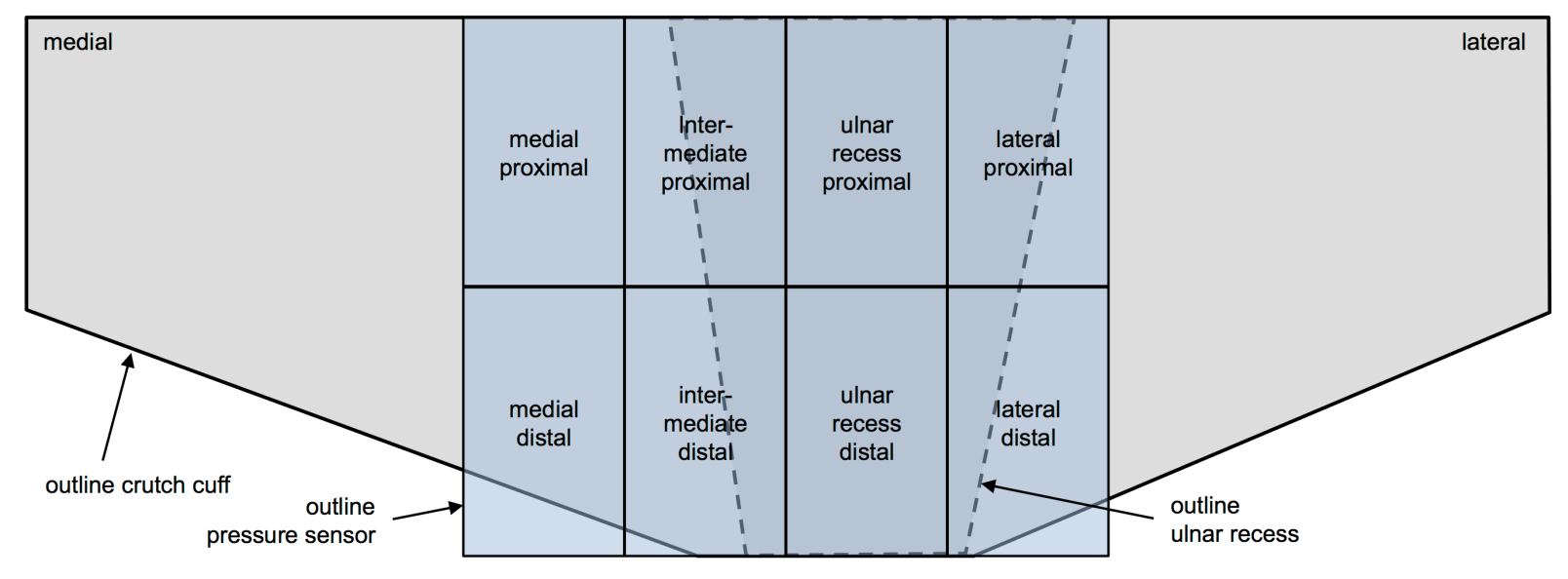

Figure 2 


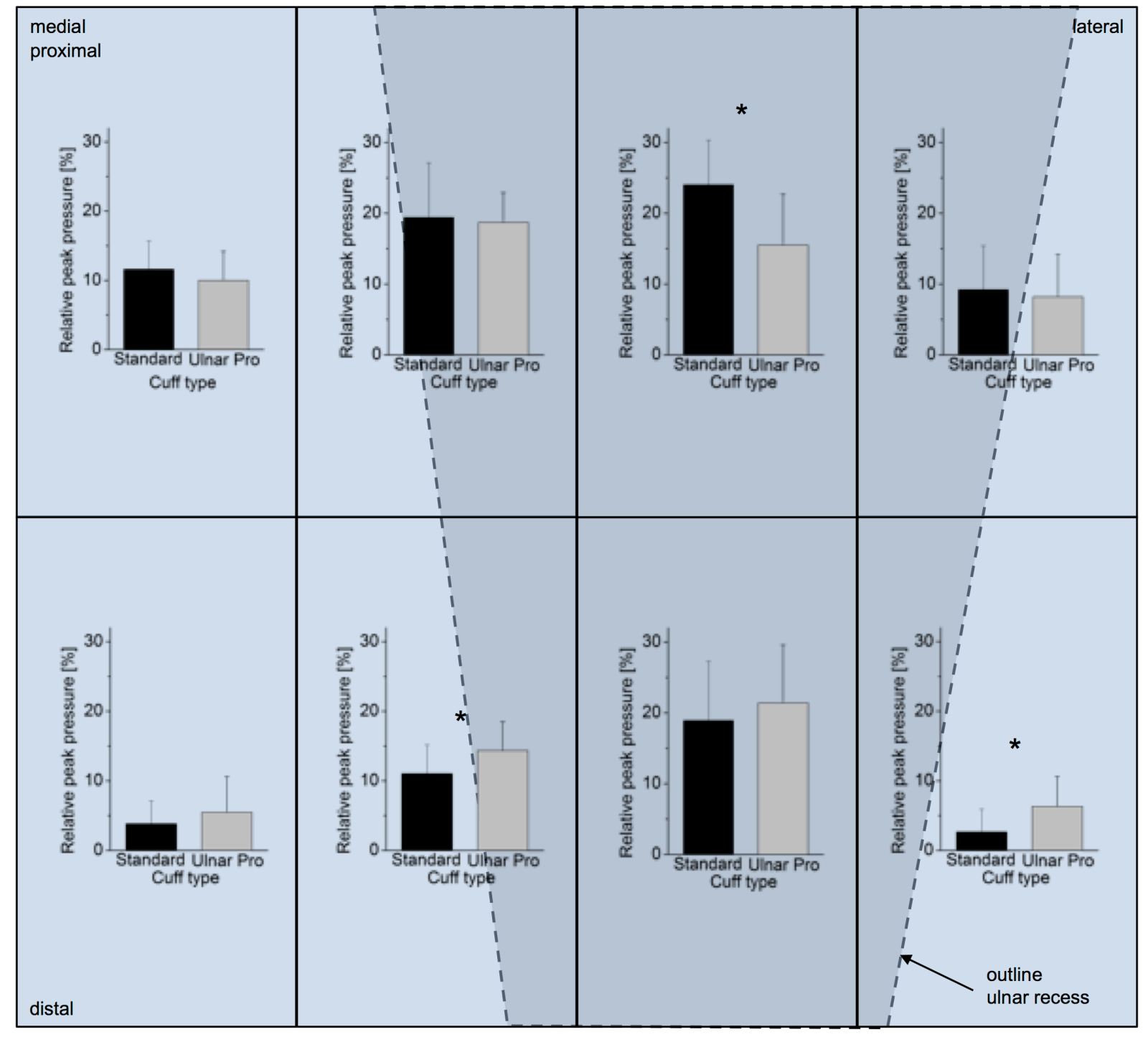

Figure 3 


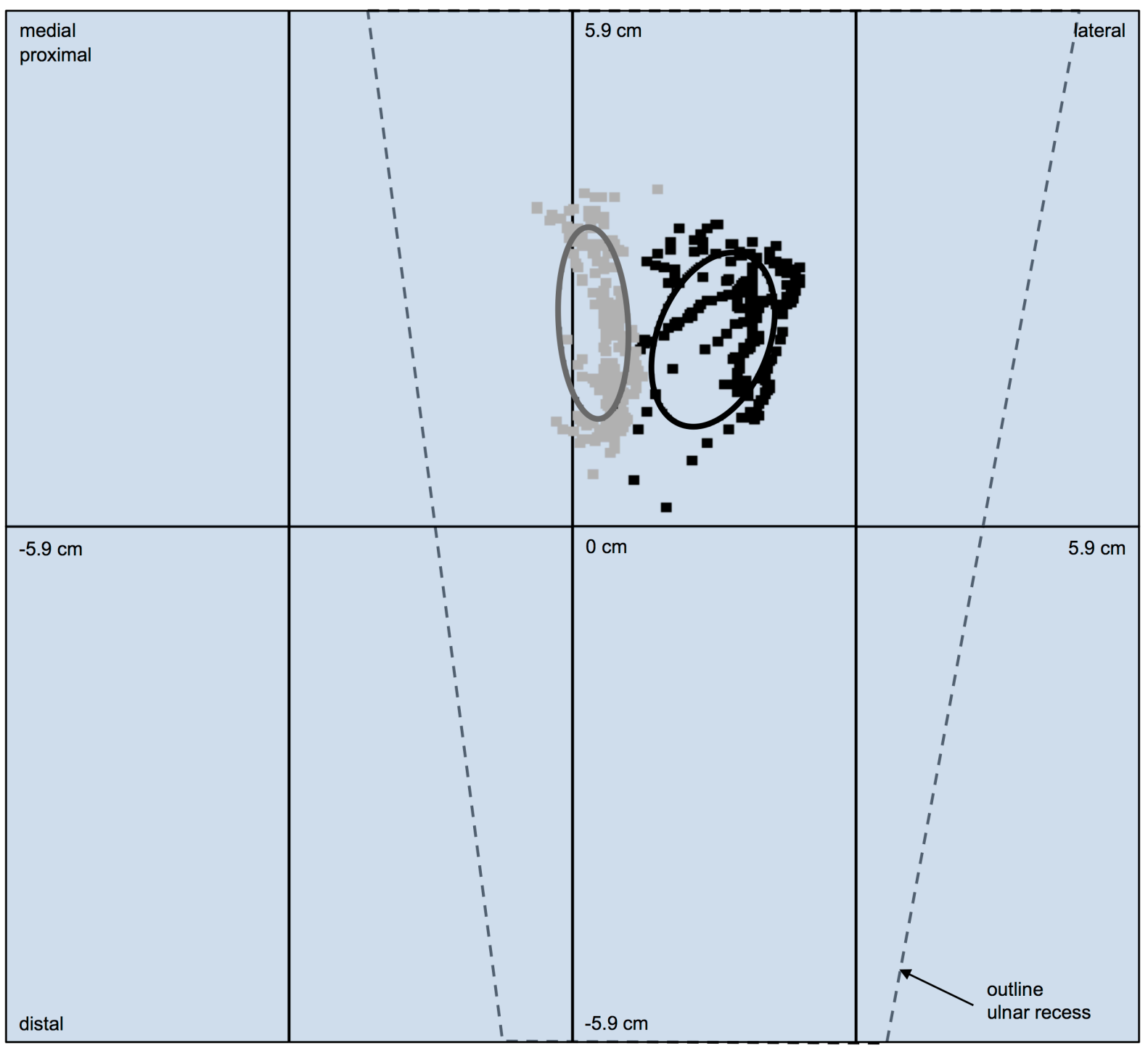

Figure 4 

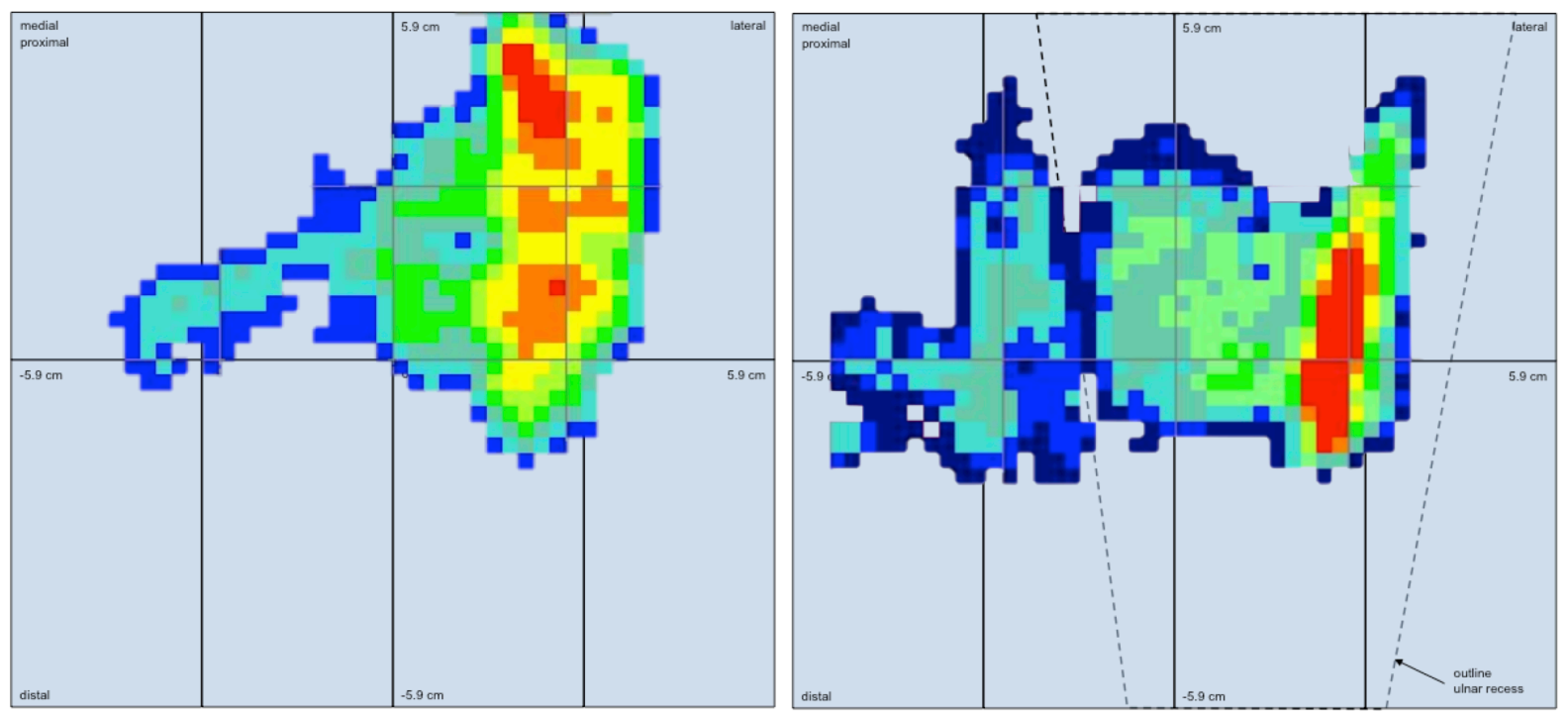

Figure 5 